\section{Percutaneous bone biopsy using a flat-panel cone beam computed tomography virtual navigation system}

\author{
Juan-Fang Liu, MD, PhD, De-Chao Jiao, MD, PhD, \\ Jian-Zhuang Ren, $M D$, PhD, Wen-Guang Zhang, $M D, P h D$, \\ Xin-Wei Han, MD, PhD.
}

\begin{abstract}
Objectives: To assess the clinical role of flat-panel cone beam CT (CBCT) in performing percutaneous needle biopsy (PNB) of bone lesions. Flat-panel cone beam CT systems have been used as a guidance tool for performing percutaneous biopsy. Real-time fluoroscopy and virtual navigation systems help simplify needle path planning and shorten procedure times.
\end{abstract}

Methods: From March 2012 to March 2016, 80 patients with 80 bone lesions were retrospectively enrolled in the study in Zhengzhou city of China. Technical success, diagnostic accuracy, puncture performance, procedure time, complications, and effective radiation exposure were calculated.

Results: All biopsies were technically successful $(100 \%)$. Sufficient tissue for histopathological analysis was obtained in 75 of 80 cases. The sensitivity of PNB of bone lesions was $95.5 \%$, the specificity was $83.3 \%$, and accuracy was $93.7 \%$. The mean scoring of puncture performance was $3.9 \pm 1.3$. The mean total procedure time was $13.20 \pm 4.4 \mathrm{~min}$, resulting in a mean exposure dose of $11.3 \pm 5.1 \mathrm{mSv}$. The complication rate was $8.7 \%$.

Conclusion: Percutaneous bone biopsy using CBCT is a safe and effective method that simplifies needle path planning and shortens procedure times.

Saudi Med J 2018; Vol. 39 (5): 519-523 doi: 10.15537/smj.2018.5.21872

A ccurately diagnosing bone tumors is crucial because appropriate treatment varies according to pathological type. ${ }^{1}$ However, current imaging techniques are not yet able to accurately diagnose bone lesions, and surgical biopsies are costly and traumatic to patients. Currently, ultrasound or computed tomography (CT)-guided percutaneous needle biopsy (PNB) is becoming popular for its convenience and accuracy. ${ }^{2-4}$ However, conventional CT guidance has limitations given its lack of real-time monitoring and gantry tilting for a more accessible needle pathway to the target lesion. Along with the development of cone beam CT (CBCT), a novel technique for PNB has recently emerged. It combines advanced real-time fluoroscopy and virtual navigation functions, which simplifies needle path planning and shortens procedure times, and reduces exposure dose and complications, while enhancing diagnostic accuracy. ${ }^{5}$ Cone beam CT-guided PNB of mediastinal lesions, pulmonary nodules, lumbo-sacrum, and cervical bone have been reported. ${ }^{6-8}$ The purpose of this study was to evaluate the diagnostic accuracy of CBCT-guided PNB in bone tumors.

Methods. Given the retrospective nature of the study and the use of anonymized data, requirements for informed consent were waived. This study was conducted in accordance with Declaration of Helsinki. The inclusion criteria were patients being able to tolerate this mini-invasive procedure. The exclusion criteria were patients who combined with severe cardiopulmonary failure or coagulation dysfunction. After institutional review board, approval was obtained from the authors' hospital, a total of 80 patients ( 43 male, 37 female; mean age $49.00 \pm 12.21$ years, range $15-77$ years) with 80 lesions were retrospectively enrolled from March 2012 to March 2016 from the authors' department (Table 1). All patients underwent enhanced CT or MRI to confirm musculoskeletal lesions. Before the biopsy procedure, a history of bleeding diathesis, coagulation parameters, including platelet count, partial thromboplastin time and prothrombin time, were evaluated. After the operation, all patients were asked to stay at the hospital for 24 hours.

All PNBs were performed under local anesthesia in supine or prone positions according to the location of the lesion and the large vessels, as well as the surrounding structures. A 13- or 16-gauge needle, and 16-gauge semi-automatic biopsy gun (Quick-Core, Cook Medical Inc, Bloomington, IN, USA), were used. Before biopsy, a pre-procedural scan under CBCT was performed to identify the safest and best access to the target lesion, avoiding obstruction and reducing distance. After each 8 DR scan, CBCT images were transferred to a detailed medical imaging workstation,

Disclosure. Authors have no conflict of interests, and the work was not supported or funded by any drug company. 
Table 1 - Lesion characteristics and procedure records.

\begin{tabular}{|c|c|c|}
\hline \multirow{2}{*}{ Data collection } & \multicolumn{2}{|c|}{ Value } \\
\hline & $\mathbf{n}$ & $(\%)$ \\
\hline Total number of patients & \multicolumn{2}{|l|}{80} \\
\hline Male/ female & \multicolumn{2}{|c|}{$43 / 37$} \\
\hline Average age(year) & \multicolumn{2}{|c|}{$49 \pm 12.21(15-87)$} \\
\hline Max diameter of lesion $(\mathrm{cm})$ & \multicolumn{2}{|c|}{$3.7 \pm 1.6(1.5-8)$} \\
\hline Depth (form skin to lesion) $(\mathrm{cm})$ & \multicolumn{2}{|c|}{$4.5 \pm 2.2(2-9)$} \\
\hline \multicolumn{3}{|l|}{ Location of lesion } \\
\hline Vertebral & 32 & $(40.0)$ \\
\hline Lumber spine & 14 & $(17.5)$ \\
\hline Thoracic spine & 11 & $(13.7)$ \\
\hline Cervical spine & 7 & $(8.7)$ \\
\hline Leg & 13 & $(16.2)$ \\
\hline Pelvis & 11 & $(13.7)$ \\
\hline Sterum & 10 & $(12.5)$ \\
\hline Rib & 8 & $(10.0)$ \\
\hline Arm & 6 & $(7.5)$ \\
\hline \multicolumn{3}{|l|}{ Lesion feature } \\
\hline Osteolytic & 70 & $(87.5)$ \\
\hline Sclerotic & 8 & $(10.0)$ \\
\hline Mixed & 2 & $(2.5)$ \\
\hline \multicolumn{3}{|l|}{ Patient position } \\
\hline Supine & 51 & $(63.7)$ \\
\hline Prone & 29 & $(36.2)$ \\
\hline Number of biopsy & $2.4 \pm 1$ & $2(1-6)$ \\
\hline Number of CBCT acquisition & $3.0 \pm 1$ & $1(2-8)$ \\
\hline Score of puncture performance & $3.9 \pm 1$ & $3(3-5)$ \\
\hline Procedure time (min) & $13.20 \pm 4$ & $4(8-32)$ \\
\hline Effective dose (mSv) & $11.3 \pm 5.1$ & $(7.1-20.3)$ \\
\hline
\end{tabular}

where the most safe and effective skin puncture points and needle pathways could be calculated. After the virtual navigation system was adjusted, the needle was advanced along the planned path into the target lesions, with the help of x-ray film and virtual color overlap (Figures 1 \& 2). Percutaneous needle biopsys were performed by one highly experienced bone radiologist and under the supervision of one chest radiologist (Han XW, 30 years' experience in image-guided PNB). After biopsy, post-procedure CT images were acquired to identify procedure-related complications.

The puncture time was defined as the time between the first scan and the completion of the procedure. Technical success was defined as appropriate localization of the biopsy needle within target nodules on procedural CBCT images and adequate tissue sampling on visual inspection. The puncture performance level was assessed using the following method: unsuccessful puncture scored 1; successful puncture with $>4$ needle repositionings or reinsertions (adjustments) scored 2; with 3-4 adjustments scored 3; with 1-2 adjustments scored 4; and without needle adjustment scored 5.

Benign PNB results were considered to be true negative if the target lesion did not receive any treatment, or the size of the lesion remained stable or reduced by $\geq 20 \%$ in the CT examination 6 months later, or surgical resection specimens exhibited certain benign diseases such as fibrosteoma or chondroma. Conversely, if the PNB result was benign and surgical excision of the specimen was malignant, this condition was termed false negative. Percutaneous needle biopsyrelated complications consisted of small hematoma(s) and mild pain.

Statistical analysis. All data analyses were performed using Excel 2010 (Microsoft Corporation, Redmond, WA, USA) and the Statistical Package for the Social Science (SPSS) version 19.0 (IBM Corp., Armonk, NY, USA). Numerical data are reported as mean \pm standard deviation.

Results. Lesion and procedural records. A detailed description of the lesion characteristics and procedure records is summarized in Table 1 . The mean maximum lesion diameter was $3.7 \pm 2.4 \mathrm{~cm}$. Among the 80 nodules, 70 lesions were osteolytic, 8 were sclerotic, and 2 were mixed. Thirty-two lesions were vertebral, 13 were located in the leg, 11 were in the pelvis, 10 in the sternum, 8 in the ribs, and 6 in the arm. The mean number of biopsies was $2.4 \pm 1.2$ and CBCT acquisitions were $3.0 \pm 1.1$. The mean total procedure time was $13.2 \pm 4.4 \mathrm{~min}$. During the entire process, the mean effective exposure dose was $11.3 \pm 5.1 \mathrm{mSv}$.

Pathological results. Detailed pathological results are summarized in Table 2. Among the 80 nodules, 65 were malignant, 10 were benign, and 5 lacked sufficient tissue for diagnosis. The 65 malignant tumors consisted of 19 osteosarcoma, 15 chondrosarcoma, 4 leiomyosarcoma, 3 angiosarcoma, 6 fibrosarcoma, and 18 metastatic tumor. The final diagnosis of malignancy was made on the basis of surgical pathology $(n=36)$, specific malignant biopsy results $(\mathrm{n}=8)$, and symptoms of advanced stage or distant metastasis receiving local radiation and chemotherapy $(n=21)$. Among the benign tumors, 5 were giant cell tumors, 2 were fibromas, and 3 were aneurysmal bone cysts. Diagnosis of the benign tumor was made according to surgical pathology $(n=4)$, follow-up observation $(n=6)$. These lesions did not receive any treatment; however, the size of the lesion remained stable or was reduced by $\geq 20 \%$ in the CT examination 6 months later. Accurate pathological diagnosis was not possible in the remaining 5 lesions because of inadequate or unspecific tissue, with results 


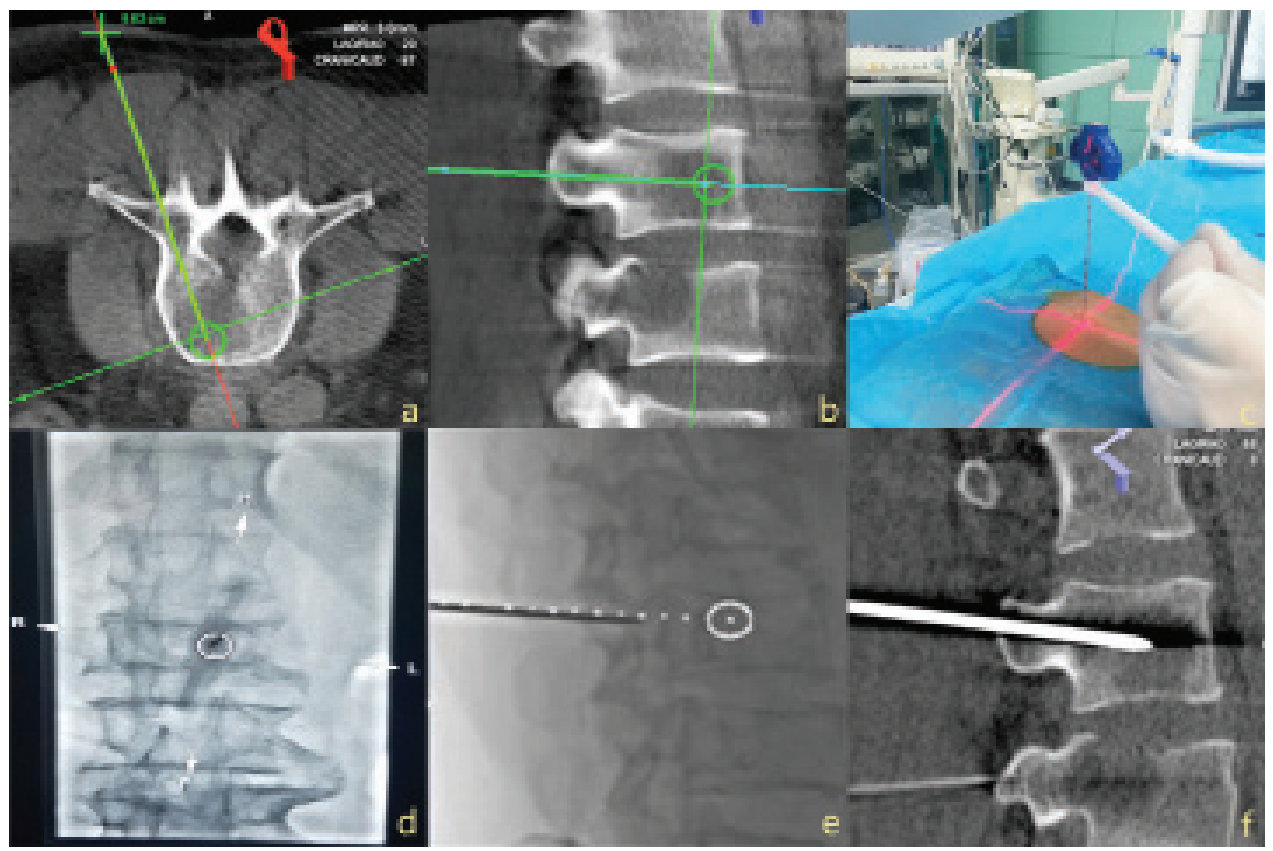

Figure 1 - The hole procedure of percutaneous biopsy of a L1 bone lesion in a 70-year-old man. A pre-procedure cone beam CT (CBCT) scan was carried out, the lesion was marked with a circle and a cross; a) axial view; b) sagittal view. Laser crosshair projected on the skin and the fixed lever placed around the needle to aid in laser alignment in the bull`s-eye view; c) The real-time fluoroscopy could help us to check the needld path; d) The planned needle path (in white) was overlaid on real-time fluoroscopy; e) The needle was aligned and inserted at the skin entry site using the bull's-eye view; f) Another CBCT was carried out to ensure the needle located within the lesion.

Table 2 - Pathologic outcomes.

\begin{tabular}{lc}
\hline Tumor & Value \\
\hline Maliganent & 65 \\
Osteosarcoma & 19 \\
Chondrosarcoma & 15 \\
Leiomyosarcoma & 4 \\
Angiosarcoma & 3 \\
Fibrosarcoma & 6 \\
Metastatic tumor & 36 \\
Benign & 10 \\
Giant cell tumour & 5 \\
Fibromas & 2 \\
Aneurysmal bone cyst. & 3 \\
Non specific or insufficient tissue & 5 \\
\hline
\end{tabular}

exhibiting necrotic bone and blood clot. To verify the characterization of these bone lesions, surgical bone biopsy was performed several days after PNB. The pathology results revealed that 3 of 5 were malignant ( 2 angiosarcoma, 1 leiomyosarcoma), the remaining 2 were benign (aneurysmal). The final correct diagnosis rate was $93.7 \%$, and the sensitivity of $\mathrm{PNB}$ of bone lesions was $95.5 \%$, specificity was $83.3 \%$, and accuracy was $93.7 \%$.
Complications included small hematoma and mild pain, occurring in 7 of $80(8.7 \%)$ procedures. The symptoms disappeared after local compression was applied and pain relief drugs were administered within 24 h (Table 3).

Discussion. Percutaneous needle biopsy is conventionally performed under CT or x-ray fluoroscopic guidance, which has been shown to be safe, accurate, and effective in targeting lesions with high diagnostic yield. ${ }^{9,10}$ However, these imaging techniques have many drawbacks including lack of real-time surveillance or 3-dimensional imaging.

Cone beam CT technologies can offer excellent visualization of the target lesion and surrounding tissue, provide a virtual pathway for needle approach to the target lesion, and facilitate navigation of the needle into the target. Cone beam CT-guided PNB has been applied in many areas for its high diagnostic yield, short total procedure time, low exposure dose, and minimal rate of complications in mediastinal lesions, pulmonary nodules, the lumbosacrum, cervical bone, and adrenals. ${ }^{11}$

Our study demonstrates that the technical success rate of $\mathrm{PNB}$ under this $\mathrm{CBCT}$ virtual navigation system 


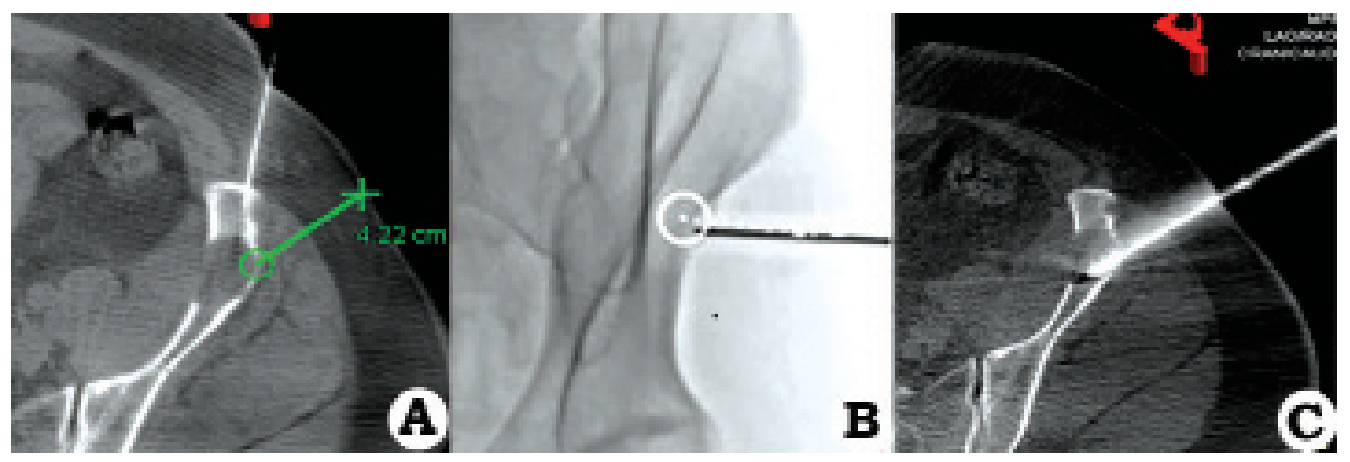

Figure 2 - Percutaneous biopsy of the left iliac bone lesion in a 55-year-old woman. a) Located the shortest distance before the procedure in the sagittal plane. b) The planned needle path (in white) was overlaid on real-time fluoroscopy. c) An updated cone beam CT (CBCT) acquired with collimated field of view confirmed the placement of the needle near or within the lesion in axial plane. (Please refer to this Figure in the Text)

Table 3 - The accuracy and complication rate of PNB procedure.

\begin{tabular}{lc}
\hline Indicator & Value \\
\hline Technical success rate (\%) & 100 \\
Sensitivity (\%) & 95.5 \\
Specificity (\%) & 83.3 \\
Accuracy (\%) & 93.7 \\
Subcutaneous hematoma & 3 \\
Pain & 2 \\
Complication rate (\%) & 6.25 \\
\hline
\end{tabular}

PNB - percutaneous needle biopsy

was $100.0 \%$, which corresponds to the rate reported by Tselikas et $\mathrm{al}^{12}$ who investigated bone biopsy under CT and CBCT (both 100\%). In our study, sufficient tissue for histopathological analysis was obtained in $93.7 \%$ of lesions. The mean total procedure time was $13.20 \pm 4.4 \mathrm{~min}$ (range $8-32 \mathrm{~min}$ ), which was in sharp contrast to the median puncture time of $32-91 \mathrm{~min}$ using CT-guidance reported in other studies. ${ }^{13}$

The effective mean exposure dose was $11.3 \pm 5.1$ $\mathrm{mSv}$, which is lower than the dose for musculoskeletal procedures (14.7 $\mathrm{mSv}-19.2 \mathrm{mSv}$ ) reported before. In our study, no serious complications occurred, either vascular or nerve injuries. There were no tumor recurrences along any biopsy pathway, only small complications such as hematomas and mild pain.

A major drawback of our study was its retrospective design. Additionally, the limited number of certain patient subgroups, such as those with primary or metastasizing tumors. However, our investigation was an observational research project, and we lacked comparative data. Finally, the resolution ratio of CBCT was lower than spiral CT with high-resolution. Motion artifacts produced by respiration or slight body movement cannot be ignored.
In conclusion, this study illustrates the promising potential of CBCT virtual navigation systems to diagnose bone lesions. It is now a better choice to perform bone biopsies under FP-CBCT guidance considering its safety and validity, and lower radiation exposure. We expect that with increased experience, more task-specific protocols and larger sample sizes, the effective radiation dose and procedure times for software-assisted cases will decrease even more in the near future.

\section{Received 14th December 2017. Accepted 7th March 2018.}

From the Department of Interventional Radiology, The First Affiliated Hospital of Zhengzhou University, Zhengzhou, Henan, People's Republic of China. Address correspondence and reprints request to: Dr. Xinwei Han, Department of Interventional Radiology, The First Affiliated Hospital of Zhengzhou University, Zhengzhou, Henan, Peoples Republic of China. E-mail: hanxinwei2006@163.com

ORCID ID: orcid.org/0000-0003-0564-7949.

\section{References}

1. Redondo A, Bagué S, Bernabeu D, Ortiz-Cruz E, Valverde C, Alvarez R, et al. Malignant bone tumors (other than Ewing's): clinical practice guidelines for diagnosis, treatment and follow-up by Spanish Group for Research on Sarcomas (GEIS). Cancer Chemother Pharmacol 2017; 80: 1113-1131.

2. Garnon J, Koch G, Tsoumakidou G. Ultrasound-guided biopsies of bone lesions without cortical disruption using fusion imaging and needle tracking: proof of concept. Cardiovasc Intervent Radiol 2017; 40: 1267-1273.

3. Cox M, Pukenas B, Poplawski M, Bress A, Deely D, Flanders A, et al. CT-guided cervical bone biopsy in 43 patients: diagnostic yield and safety at two large tertiary care hospitals. Acad Radiol 2016; 23 : $1372-1375$.

4. Monfardini L, Preda L, Aurilio G, Rizzo S, Bagnardi V, Renne G, et al. CT-guided bone biopsy in cancer patients with suspected bone metastases: retrospective review of 308 procedures. Radiol Med 2014; 119: 852-860.

5. Abbassy MA, Sabban HM, Hassan AH, Zawawi KH. Evaluation of mini-implant sites in the posterior maxilla using traditional radiographs and cone-beam computed tomography. Saudi Med J 2015; 36: 1336-1341. 
6. Jiao D, Huang K, Wu G, Wang Y, Han X. Flat detector cone-beam CT-guided percutaneous needle biopsy of mediastinal lesions: preliminary experience. Radiol Med 2016; 121: 769-779.

7. Jiao DC, Li ZM, Yuan HF, Zhang QH, Ren JZ, Zhou PL, et al. Flat detector C-arm CT-guidance system in performing percutaneous transthoracic needle biopsy of small $(\leq 3 \mathrm{~cm})$ pulmonary lesions. Acta Radiol 2016; 57: 677-683.

8. Matsumoto T, Mine T, Hayashi T, Kamono M, Taoda A, Higaki $\mathrm{M}$, et al. CT Fluoroscopy-guided trans-sacral intervertebral drainage for pyogenic spondylodiscitis at the lumbosacral junction. Cardiovasc Intervent Radiol 2017; 40: 125-129.

9. Maciel MJ, Tyng CJ, Barbosa PN, Bitencourt AG, Matushita Junior JP, Zurstrassen CE, et al. Computed tomography-guided percutaneous biopsy of bone lesions: rate of diagnostic success and complications. Radiol Bras 2014; 47: 269-274.
10. Kiatisevi P, Thanakit V, Sukunthanak B, Boonthatip M, Bumrungchart S, Witoonchart K. Computed tomographyguided core needle biopsy versus incisional biopsy in diagnosing musculoskeletal lesions. J Orthop Surg 2013; 21: 204-208.

11. Jiao D, Xie N, Wu G, Ren J, Han X. C-arm cone-beam computed tomography with stereotactic needle guidance for percutaneous adrenal biopsy: initial experience. Acta Radiol 2017; 58: 617-624.

12. Tselikas L, Joskin J, Roquet F, Farouil G, Dreuil S, Hakimé A. Percutaneous bone biopsies: comparison between flat-panel cone-beam CT and CT-scan guidance. Cardiovasc Intervent Radiol 2015; 38: 167-176.

13. Shellikeri S, Setser RM, Hwang TJ, Srinivasan A, Krishnamurthy G, Vatsky S, et al. Real-time fluoroscopic needle guidance in the interventional radiology suite using navigational software for percutaneous bone biopsies in children. Pediatr Radiol 2017; 47: 963-973. 